\title{
The Epidemiology of Hip Fracture among Subjects with Pyogenic Liver Abscess (PLA): A Nationwide Population-Based Study
}

\author{
Chieh-Cheng Hsu $\left(\mathbb{D},{ }^{1}\right.$ Jih-Yang Ko $\mathbb{D}^{1},{ }^{1,2}$ Cheng-Li Lin $\mathbb{D}^{\circ},{ }^{3}$ Horng-Chaung Hsu, ${ }^{4,5}$ \\ Hsien-Te Chen, ${ }^{5,6,7}$ Che-Chen Lin, ${ }^{3}$ and Shu-Jui Kuo $\mathbb{D}^{4,5}$ \\ ${ }^{1}$ Department of Orthopedic Surgery, Kaohsiung Chang Gung Memorial Hospital and Chang Gung University College of Medicine, \\ Kaohsiung, Taiwan \\ ${ }^{2}$ Center for Shockwave Medicine and Tissue Engineering, Kaohsiung Chang Gung Memorial Hospital, Kaohsiung, Taiwan \\ ${ }^{3}$ Management Office for Health Data, China Medical University Hospital, Taichung, Taiwan \\ ${ }^{4}$ School of Medicine, China Medical University, Taichung, Taiwan \\ ${ }^{5}$ Department of Orthopedic Surgery, China Medical University Hospital, Taichung, Taiwan \\ ${ }^{6}$ Department of Sports Medicine, College of Health Care, China Medical University, Taichung, Taiwan \\ ${ }^{7}$ Spine Center, China Medical University Hospital, China Medical University, Taichung, Taiwan
}

Correspondence should be addressed to Shu-Jui Kuo; b90401073@gmail.com

Received 7 October 2019; Accepted 28 November 2019; Published 12 February 2020

Academic Editor: Paolo Muratori

Copyright ( $) 2020$ Chieh-Cheng Hsu et al. This is an open access article distributed under the Creative Commons Attribution License, which permits unrestricted use, distribution, and reproduction in any medium, provided the original work is properly cited.

\begin{abstract}
Pyogenic liver abscess (PLA) is a potentially fatal disease that can stimulate prominent systemic inflammation. Osteoporotic hip fracture is a major complication of systemic inflammation. This study tried to determine the epidemiology of hip fractures among PLA patients. All subjects admitted due to PLA during 1999 2010 were assessed, excluding the subjects with a history of high energy trauma, malignancy, and previous hip fracture. We matched the control subjects to PLA patients according to age, gender, and the coding of osteoporosis by $1: 4$ ratio. The PLA patients had a 1.17-fold risk of hip fracture than the controls $(\mathrm{aHR}=1.17$, 95\% CI $=1.07-1.29$ ) after adjusting for gender, age, and comorbidities. Considering death as the competing event of suicide, the PLA patients had 1.10-fold suicide risk $(\mathrm{aHR}=1.10,95 \% \mathrm{CI}: 1.00-1.21)$ than the control subjects under the competing risks regression model. The cumulative incidence of hip fracture was higher in the PLA cohort (log-rank test, $p<0.001)$. When compared to the controls, the fracture risk was 18.4-fold $(\mathrm{aHR}=18.4,95 \% \mathrm{CI}=13.0-26.1)$ for the PLA patients admitted 2-3 times per year and 46.0-fold $(\mathrm{aHR}=46.0,95 \% \mathrm{CI}=31.2-67.8)$ for the PLA patients admitted $\geqq 4$ times per year. The impact of PLA is more prominent among the subjects aged $<45$ years $(\mathrm{aHR}=2.81,95 \% \mathrm{CI}=1.42-5.56)$. Preventive measures for hip fracture might be warranted for PLA patients.
\end{abstract}

\section{Introduction}

Pyogenic liver abscess (PLA) is a potentially lethal disease with the mortality rate of up to $19 \%$ [1]. PLA can be seen in the subjects with diabetes, existing hepatobiliary disease, and in individuals who have undergone invasive procedures [2]. In recent years, the incidence of PLA has surged persistently all over the world. In the United States, the incidence of PLA has grown from 2.7 per 100,000 individuals in 1994 to 4.1 per 100,000 individuals in 2005 [3]. In Taiwan, the prevalence was higher than that in the United States, increasing from
10.83 per 100,000 individuals in 2000 to 15.45 per 100,000 individuals in 2011 [4]. However, the long-term sequelae among the survivors of PLA are not completely understood at present. The increasing population of PLA, as well as the PLA survivors, underscores the importance of identifying the long-term sequelae of PLA. The use of oral corticosteroids is significantly associated with an increased risk of developing PLA with a dose-dependent effect $[5,6]$.

Osteoporosis is the major sequelae under systemic inflammation, which can be explained by the perspective of altered energy metabolism, evolution, and immunologic and 
neuroendocrine factors [7]. Osteoporotic hip fractures account for one-fourth of geriatric fractures necessitating hospitalization with the mortality rate reaching $20 \%$ [8].

Osteoporotic fractures are prevalent among individuals with hepatobiliary diseases, including primary biliary cirrhosis, chronic hepatitis $\mathrm{C}$ infection, or primary sclerosing cholangitis [9]. Recently, cholangitis has been recognized to be associated with a higher risk of hip fracture [8]. Despite the surging prevalence of PLA and the considerable mortality rate after hip fracture, the epidemiologic information of hip fracture among PLA patients is not determined at present. In our study, we want to investigate the pertinent epidemiology of hip fracture among the individuals with PLA history.

\section{Materials and Methods}

2.1. Data Source. The National Health Insurance (NHI) program was established by the Taiwanese government in 1995. It is single-payer based and nearly $99.9 \%$ Taiwanese citizens were enrolled. The database, named National Health Insurance Research Database (NHIRD), was released by the National Health Research Institute (NHRI) and contained all health claim data from Taiwan NHI. The history of disease diagnosis was obtained from the inpatient files under the coding system of the International Classification of Disease, Ninth Revision, Clinical Modification (ICD-9-CM). To protect the privacy of the subjects, the initial identification number of each individual was removed and replaced by the scrambled random number. Our study was approved by the Research Ethics Committee of China Medical University (CMUH-104-REC2-115-R3).

2.2. Study Population. This population-based cohort study tried to investigate the epidemiology of hip fracture among PLA patients. All of the subjects admitted with the de novo coding of PLA (ICD-9-CM code: 572.0) between January 1999 and December 2010 were all recruited for assessment. The date when the diagnosis of PLA was initially coded was defined as the index date. The control subjects without PLA coding were matched to the PLA patients according to gender, age (every 5 years), and the coding of osteoporosis (ICD-9-CM 733.0 and 733.1) by $1: 4$ ratio and recruited at the index date. The individuals with high energy trauma (presence of E coding) and cancer (ICD-9-CM 140-208) at the index date and the follow-up period and with the history of a previous hip fracture (ICD-9-CM 820) at the index date were excluded for both groups [8]. All of the subjects were followed until the withdrawal from the insurance, the occurrence of hip fracture, or until December 31, 2011.

The comorbidities analyzed in our study included liver cirrhosis, coronary artery disease (CAD), diabetes (DM), epilepsy, hypertension, stroke, and end-stage renal disease.

2.3. Statistical Analysis. We used the two-sample $t$-test for the comparison of continuous variables and the chi-square test for categorical variables. The Kaplan-Meier method was utilized to plot the survival curves for the two cohorts with the log-rank test chosen to express the significance of difference. The hazard ratios (HRs), adjusted hazard ratios (aHR), and 95\% confidence intervals (CIs) were presented by using crude and adjusted Cox proportional hazard models to compare the risk of hip fracture in both cohorts.

The proportional hazard model assumption was also examined using a test of scaled Schoenfeld residuals. In the model evaluating the hip fracture risk throughout the overall follow-up period, the results of the test revealed a significant relationship between Schoenfeld residuals for PLA and follow-up time, suggesting that the proportionality assumption was conformed $(p$ value $=0.06)$. The subhazard ratio (SHR) was also calculated after further controlling for the competing risk of deaths using the Fine and Gray method as a sensitivity analysis. All statistical analyses were performed using SAS statistical software, version 9.4 (SAS Institute Inc., Cary, NC). The figure of the cumulative incidence curve was plotted by $\mathrm{R}$ software. The significant criteria were set below 0.05 for the $p$ value of the two-sided testing [9].

\section{Results}

There were 25530 patients in the PLA cohort and 102120 subjects in the control cohort in our study (Table 1). In the study population, $63.0 \%$ were male. The mean age of the PLA cohort and the control cohort was $59.4 \pm 14.6$ and $59.3 \pm 14.6$ years, respectively. The composition of gender, age, and osteoporosis was homogenous between the two groups. The prevalence of liver cirrhosis, CAD, diabetes, epilepsy, hypertension, stroke, ESRD, and COPD was significantly higher in the PLA cohort (all $p<0.001$ ).

The incidence of hip fracture was 44.8 per 10000 personyears in the PLA cohort and 33.7 per 10000 person-years in the control cohort (Table 2). The patients with PLA had a 1.17-fold fracture risk than the subjects without PLA $(\mathrm{aHR}=1.17,95 \% \mathrm{CI}=1.07-1.29)$ after adjusting for gender, age, and all comorbidities. The cumulative incidence of hip fracture was significantly higher in the PLA cohort (log-rank test $p<0.01$ ) (Figure 1). Considering death as the competing event of suicide, the PLA patients had 1.10-fold suicide risk $(\mathrm{aHR}=1.10,95 \% \mathrm{CI}: 1.00-1.21)$ than the control subjects under the competing risks regression model (Table 3 ). The risk of hip fracture was significantly higher among subjects from 45 to 64 years of age $(\mathrm{aHR}=3.48,95 \% \mathrm{CI}=2.53-4.78)$ and $\geqq 65$ years old $(\mathrm{aHR}=21.4,95 \% \mathrm{CI}=15.7-29.1)$. The male subjects had lower risk of hip fracture $(\mathrm{aHR}=0.65,95 \%$ $\mathrm{CI}=0.60-0.70)$ than the female subjects. The patients with osteoporosis ( $\mathrm{aHR}=1.57,95 \% \mathrm{CI}=1.32-1.85)$, liver cirrhosis $(\mathrm{aHR}=1.80,95 \% \mathrm{CI}=1.37-2.37), \mathrm{DM} \quad(\mathrm{aHR}=1.38$, 95\% CI $=1.26-1.50)$, hypertension $\quad(\mathrm{aHR}=1.23, \quad 95 \%$ $\mathrm{CI}=1.11-1.36)$, stroke $(\mathrm{aHR}=1.64,95 \% \mathrm{CI}=1.46-1.85)$, ESRD $\quad(\mathrm{aHR}=2.05,95 \% \mathrm{CI}=1.52-2.75)$, and COPD $(\mathrm{aHR}=1.71,95 \% \mathrm{CI}=1.48-1.97)$ had higher risk of hip fracture (Table 2). Table 4 demonstrated the incidence and adjusted hazard ratio of hip fracture stratified by the admission frequency under the coding of PLA. The risk of hip fracture among the subjects who were admitted 2-3 times per year was 18.4-fold $(\mathrm{aHR}=18.4,95 \% \mathrm{CI}=13.0-26.1)$ 
TABLE 1: Comparison of baseline demographic profiles and history of comorbidities between the control and PLA cohorts.

\begin{tabular}{|c|c|c|c|}
\hline Variable & Control cohort $N=102120(\%)$ & PLA cohort $N=25530(\%)$ & $p$ value \\
\hline Age, year (SD) & $59.3(14.6)$ & $59.4(14.6)$ & 0.29 \\
\hline Sex & & & $>0.99$ \\
\hline Female & $37752(37.0)$ & $9438(37.0)$ & \\
\hline Male & $64368(63.0)$ & $16092(63.0)$ & \\
\hline \multicolumn{4}{|l|}{ Comorbidities } \\
\hline Osteoporosis & $2164(2.1)$ & $541(2.1)$ & $>0.99$ \\
\hline Liver cirrhosis & $706(0.7)$ & $743(2.9)$ & $<0.01$ \\
\hline $\mathrm{CAD}$ & $6138(6.0)$ & $2554(10.0)$ & $<0.01$ \\
\hline $\mathrm{DM}$ & $14748(14.4)$ & $10264(40.2)$ & $<0.01$ \\
\hline Epilepsy & $304(0.3)$ & $131(0.5)$ & $<0.01$ \\
\hline Hypertension & $12459(12.2)$ & $7421(29.1)$ & $<0.01$ \\
\hline Stroke & $5477(5.4)$ & $2084(8.2)$ & $<0.01$ \\
\hline ESRD & $532(0.5)$ & $594(2.3)$ & $<0.01$ \\
\hline COPD & $3056(3.0)$ & $1293(5.1)$ & $<0.01$ \\
\hline
\end{tabular}

PLA: pyogenic liver abscess; CAD: coronary artery disease; DM: diabetes mellitus; ESRD: end-stage renal disease; COPD: chronic obstructive pulmonary disease.

TABLE 2: Incidence and adjusted hazard ratio of hip fracture stratified by PLA, age, gender, and comorbidities.

\begin{tabular}{|c|c|c|c|c|c|}
\hline Variables & Event & PYs & Rate & Crude HR 95\% CI & Adjusted HR 95\% CI \\
\hline \multicolumn{6}{|l|}{ PLA } \\
\hline No & 2067 & 613796 & 33.7 & Ref & Ref \\
\hline Yes & 616 & 137586 & 44.8 & $1.34(1.22-1.46)$ & $1.17(1.07-1.29)$ \\
\hline \multicolumn{6}{|c|}{ Age group, years } \\
\hline$<45$ & 42 & 141361 & 2.97 & Ref & Ref \\
\hline $45-64$ & 446 & 364211 & 12.3 & $4.16(3.03-5.70)$ & $3.48(2.53-4.78)$ \\
\hline$\geqq 65$ & 2195 & 245811 & 89.3 & $31.1(22.9-42.3)$ & $21.4(15.7-29.1)$ \\
\hline \multicolumn{6}{|l|}{ Sex } \\
\hline Female & 1479 & 277483 & 53.3 & Ref & Ref \\
\hline Male & 1204 & 473900 & 25.4 & $0.48(0.44-0.51)$ & $0.65(0.60-0.70)$ \\
\hline \multicolumn{6}{|c|}{ Osteoporosis } \\
\hline No & 2518 & 739214 & 34.1 & Ref & Ref \\
\hline Yes & 165 & 12169 & 136 & $4.05(3.46-4.75)$ & $1.57(1.32-1.85)$ \\
\hline \multicolumn{6}{|c|}{ Liver cirrhosis } \\
\hline No & 2630 & 746050 & 35.3 & Ref & Ref \\
\hline Yes & 53 & 5332 & 99.4 & $2.89(2.20-3.80)$ & $1.8(1.37-2.37)$ \\
\hline \multicolumn{6}{|l|}{ CAD } \\
\hline No & 2316 & 712661 & 32.5 & Ref & Ref \\
\hline Yes & 367 & 38721 & 94.8 & $2.99(2.67-3.34)$ & $1.08(0.96-1.22)$ \\
\hline \multicolumn{6}{|l|}{ DM } \\
\hline No & 1846 & 621865 & 29.7 & Ref & Ref \\
\hline Yes & 837 & 129518 & 64.6 & $2.21(2.03-2.39)$ & $1.38(1.26-1.50)$ \\
\hline \multicolumn{6}{|l|}{ Epilepsy } \\
\hline No & 2665 & 749657 & 35.6 & Ref & Ref \\
\hline Yes & 18 & 1726 & 104 & $3.00(1.89-4.77)$ & $1.52(0.95-2.43)$ \\
\hline \multicolumn{6}{|c|}{ Hypertension } \\
\hline No & 1866 & 662023 & 28.2 & Ref & Ref \\
\hline Yes & 817 & 89360 & 91.4 & $3.36(3.09-3.65)$ & $1.23(1.11-1.36)$ \\
\hline \multicolumn{6}{|l|}{ Stroke } \\
\hline No & 2291 & 720446 & 31.8 & Ref & Ref \\
\hline Yes & 392 & 30936 & 127 & $4.13(3.71-4.60)$ & $1.64(1.46-1.85)$ \\
\hline \multicolumn{6}{|l|}{ ESRD } \\
\hline No & 2637 & 747524 & 35.3 & Ref & Ref \\
\hline Yes & 46 & 3858 & 119 & $3.48(2.60-4.66)$ & $2.05(1.52-2.75)$ \\
\hline \multicolumn{6}{|l|}{ COPD } \\
\hline No & 2445 & 734019 & 33.3 & Ref & Ref \\
\hline Yes & 238 & 17364 & 137 & $4.23(3.70-4.83)$ & $1.71(1.48-1.97)$ \\
\hline
\end{tabular}

Model adjusted for gender, age, and comorbidities. PLA: pyogenic liver abscess; PYs: person-years; rate: incidence rate per 10000 person-years; HR: hazard ratio; CI: confidence interval; CAD: coronary artery disease; DM: diabetes mellitus; ESRD: end-stage renal disease; COPD: chronic obstructive pulmonary disease. 


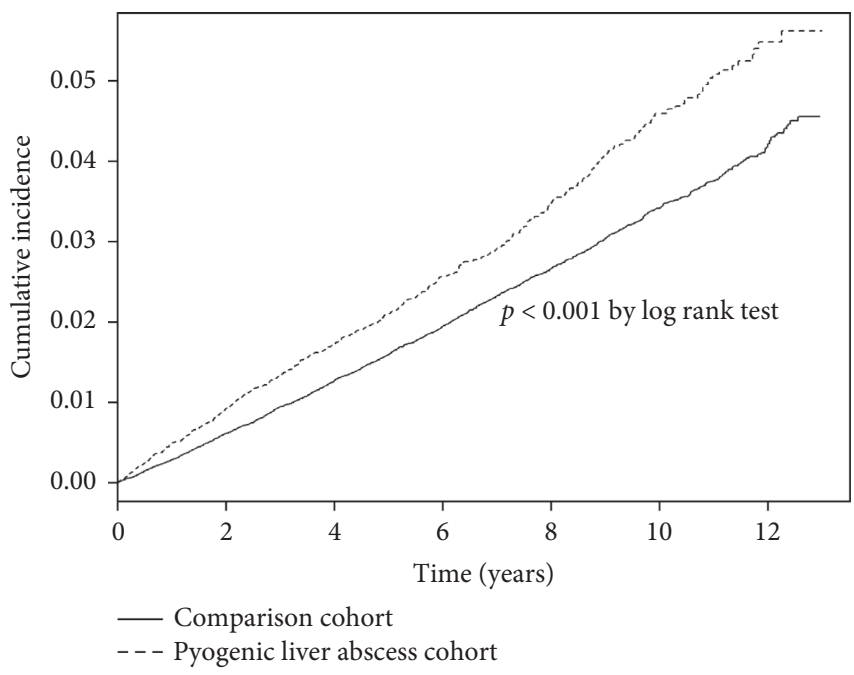

Figure 1: The Kaplan-Meier survival curves for PLA and comparison cohorts. The incidence of hip fracture with substantially higher in the PLA cohort ( $p<0.001$ by log-rank test).

TABLE 3: The subhazard ratio of hip fracture estimated through the competing risks regression model.

\begin{tabular}{lccc}
\hline \multirow{2}{*}{ Variable } & \multicolumn{2}{c}{ Pyogenic liver abscess } & \multirow{2}{*}{ N value } \\
& No & Yes & \\
\hline Crude SHR $(95 \% \mathrm{CI})$ & 1 (reference) & $1.24(1.13,1.36)$ & $<0.001$ \\
Adjusted SHR $^{\dagger}(95 \% \mathrm{CI})$ & 1 (reference) & $1.10(1.00,1.21)$ & 0.04 \\
\hline
\end{tabular}

SHR: subhazard ratio, crude SHR: relative subhazard ratio; adjusted $\mathrm{SHR}^{\dagger}$ : multivariable analysis including all factors in the univariable Cox model. ${ }^{*} p<0.05 ;{ }^{* *} p<0.01 ;{ }^{* * *} p<0.001$.

TABLE 4: Incidence of hip fracture stratified by the frequency of admission under the coding of PLA.

\begin{tabular}{lccccc}
\hline & $N$ & Event & PYs & Rate & $\begin{array}{c}\text { Adjusted HR } \\
95 \% \text { CI }\end{array}$ \\
\hline $\begin{array}{l}\text { Comparison } \\
\text { cohort }\end{array}$ & 102120 & 2067 & 613796 & 33.7 & Ref \\
$\begin{array}{l}\text { PLA cohort } \\
\quad\end{array}$ & 23869 & 544 & 137051 & 39.7 & $1.08(0.98-1.19)$ \\
$2-3$ & 564 & 35 & 378 & 927 & $18.4(13.0-26.1)$ \\
$\geq 4$ & 1097 & 37 & 157 & 2353 & $46.0(31.2-67.8)$ \\
$\quad p$ for trend & & & & & $<0.001$ \\
\hline
\end{tabular}

Model adjusted for gender, age, and comorbidities. PLA: pyogenic liver abscess; $N$ : number of subjects within the cohort; event: hip fracture numbers; PYs: person-years; rate: incidence rate per 10000 person-years; HR: hazard ratio.

compared to the control cohort. The risk of hip fracture increased to 46.0 -fold ( $\mathrm{aHR}=46.0,95 \% \mathrm{CI}=31.2-67.8)$ for the PLA patients who were admitted $\geqq 4$ times per year. The higher the admission frequency under the coding of PLA, the higher the risk of hip fracture ( $p$ for trend $<0.001$ ).

Table 5 compared the incidence and adjusted hazard ratio of hip fracture between the PLA and non-PLA cohorts stratified by gender, age, and comorbidities. PLA is associated with higher risk of hip fracture in both males $(\mathrm{aHR}=1.25,95 \% \mathrm{CI}=1.09-1.44)$ and females $(\mathrm{aHR}=1.18$,
$95 \% \mathrm{CI}=1.04-1.34)$. The impact of PLA on the occurrence of hip fracture is more prominent among the subjects aged $<45$ years old $(\mathrm{aHR}=2.81,95 \% \mathrm{CI}=1.42-5.56)$.

\section{Discussion}

In our study, we showed that the subjects with the coding of PLA had a 1.17-fold risk of suffering from hip fracture than the individuals without PLA after adjusting for age, gender, and pertinent comorbidities. We also showed that the admission frequency is positively correlated with hip fracture risk. We demonstrated that the impact of PLA on the occurrence of hip fracture is more prominent among the individuals aged $<45$ years old and among the subjects with pertinent comorbidities $(\mathrm{aHR}=1.15,95 \% \mathrm{CI}=1.03 \sim 1.28$ ). Considering death as the competing event of suicide, the PLA patients had 1.10 -fold suicide risk ( $\mathrm{aHR}=1.10,95 \%$ CI: $1.00-1.21)$ than the control subjects under the competing risks regression model.

This study is the first one demonstrating the epidemiology of hip fracture among PLA patients.

The "three-pillar theory" tried to describe the possible mechanisms of inflammatory bone loss, including disturbed energy metabolism, evolution, and immunologic and neuroendocrine factors [7]. The receptor activator of nuclear factor- $\kappa \mathrm{B}$ (RANK) is widely known as an immunologic factor and osteoclastic activator $[7,8]$. The mortality rate of osteoporotic hip fracture is as high as $20 \%$ within 1 year. We thus tried to determine the epidemiology of hip fracture in the disease entities that are capable of stimulating systemic inflammation, such as PLA. Previous studies showed that $>90 \%$ PLA patients were presented with fever and $>25 \%$ with positive bacterial culture, highlighting the phenomena that PLA can trigger substantial systemic inflammation [1]. It is worth-investigating whether the extent of PLA-induced inflammation could lead to osteoporotic hip fracture.

Hsu et al. have shown that the risk of hip fracture was substantially higher among the patients afflicted with cholangitis than that among the control subjects $(\mathrm{aHR}=1.29,95 \% \mathrm{CI}=1.03-1.61)$ in one population-based study [8]. Angulo et al. also showed that osteoporosis could be observed in $15 \%$ individuals afflicted with primary sclerosing cholangitis, and the incidence rate was 23.8 -fold than the gender and age-matched controls [10]. These results suggested the correlation between hepatobiliary inflammation and bone loss. In our study, we showed that the patients with PLA had 1.17 times the possibility of suffering from hip fractures than the matched control individuals. We also demonstrated that the possibility of hip fracture among the subjects who were admitted 2-3 times per year under the coding of PLA was 18.4-fold compared to the control cohort, and the risk increased to 46.0 -fold among the PLA patients who were admitted $\geqq 4$ times per year. These findings suggested that the higher the severity of PLA, the higher the risk of hip fracture.

Age, female gender, osteoporosis, liver cirrhosis, DM, hypertension, stroke, ESRD, and COPD were also correlated with a higher risk of hip fracture in our model. These findings inferred from our model were in keeping with the 
TABLE 5: Incidence and hazard ratio of hip fracture among the PLA and control cohorts stratified by gender and age.

\begin{tabular}{|c|c|c|c|c|c|c|c|c|}
\hline \multirow{3}{*}{ Variables } & \multicolumn{6}{|c|}{ PLA } & \multirow{3}{*}{ Crude HR $(95 \%$ CI) } & \multirow{3}{*}{ Adjusted HR (95\% CI) } \\
\hline & \multicolumn{3}{|c|}{ No $(N=102120)$} & \multicolumn{3}{|c|}{ Yes $(N=25530)$} & & \\
\hline & Event & Person-years & Rate $^{\#}$ & Event & Person-years & Rate $^{\#}$ & & \\
\hline \multicolumn{9}{|l|}{ Gender } \\
\hline Female & 1147 & 227197 & 50.5 & 332 & 50286 & 66.0 & $1.32(1.17,1.49)$ & $1.18(1.04,1.34)$ \\
\hline Male & 920 & 386600 & 23.8 & 284 & 87300 & 32.5 & $1.37(1.20,1.57)$ & $1.25(1.09,1.44)$ \\
\hline \multicolumn{9}{|l|}{ Age, years } \\
\hline$<45$ & 22 & 114174 & 1.93 & 20 & 27187 & 7.36 & $3.84(2.09,7.03)$ & $2.81(1.42,5.56)$ \\
\hline $45-64$ & 310 & 296756 & 10.5 & 136 & 67456 & 20.2 & $1.97(1.61,2.41)$ & $1.23(0.98,1.53)$ \\
\hline$\geqq 65$ & 1735 & 202867 & 85.5 & 460 & 42944 & 107.1 & $1.27(1.14,1.40)$ & $1.10(0.99,1.23)$ \\
\hline
\end{tabular}

PLA: pyogenic liver abscess; $\mathrm{HR}=$ hazard ratio, $\mathrm{CI}=$ confidence interval. PYs: person-years; rate ${ }^{\#}$ : incidence rate per 10000 person-years. Model adjusted for gender, age, and comorbidities.

previous studies, consolidating the validity of our model [8, 11].

It is intriguing that the impact of PLA on the occurrence of hip fracture is more prominent among the subjects aged $<45$ years old $(\mathrm{aHR}=2.81,95 \% \mathrm{CI}=1.42-5.56)$. We hypothesize that the subjects $<45$ years old have fewer established risk factors, such as age, ESRD, and stroke, for hip fractures, so the impact of PLA is less diluted [12, 13]. The correlation between PLA, age, and hip fracture warrants further investigation.

There are limitations to our study. First, not all of the risk factors for hip fractures could be retrieved from the NHIRD, such as body height, weight, parent hip fracture history, and the consumption of tobacco and alcohol. Second, there were substantial hip fracture patients who were coded with ICD-9-CM 820.8, the coding including transcervical and pertrochanteric fractures, in the NHIRD. As a result, we cannot differentiate the transcervical and pertrochanteric fractures among a substantial portion of hip fracture patients.

\section{Conclusions}

We showed that PLA is associated with a higher risk for hip fracture than the gender, age, and the status of osteoporosis-matched control subjects. The admission frequency is positively correlated with the risk of hip fracture. The impact of PLA is more prominent among the subjects aged $<45$ years old and the presence of comorbidities. Preventive measures for hip fracture, such as antiosteoporotic regimen, might be considered for PLA patients.

$\begin{array}{ll}\text { Abbreviations } \\ \text { PLA: } & \text { Pyogenic liver abscess } \\ \text { NHIRD: } & \text { National Health Insurance Research Database } \\ \text { NHRI: } & \text { National Health Research Institute } \\ \text { ICD-9- } & \text { International Classification of Disease, Ninth } \\ \text { CM: } & \text { Revision, Clinical Modification } \\ \text { HRs: } & \text { Hazard ratios } \\ \text { aHRs: } & \text { Adjusted hazard ratios } \\ \text { CIs: } & \text { Confidence intervals } \\ \text { RANK: } & \text { Receptor activator of nuclear factor- } \kappa \mathrm{B} .\end{array}$

\section{Data Availability}

The data used to support the findings of this study are available from the corresponding author (e-mail: b90401073@gmail.com) upon request.

\section{Conflicts of Interest}

The authors declare that they have no conflicts of interest.

\section{Acknowledgments}

This study was supported by the Ministry of Health and Welfare, Taiwan (MOHW107-TDU-B- 212-123004), China Medical University Hospital, Academia Sinica Stroke Biosignature Project (BM10701010021), MOST Clinical Trial Consortium for Stroke (MOST 106-2321-B-039-005), Tseng -Lien Lin Foundation (Taiwan), Brain Disease Foundation (Taiwan), Katsuzo and Kiyo Aoshima Memorial Funds (Japan), and Center for Shockwave Medicine and Tissue Engineering, Kaohsiung Chang Gung Memorial Hospital (CMRPG8B1301-CRRPG8B1303 and CRRPG8F0461CRRPG8F0463). These funding sources support the study to obtain the charged NHIRD data from the NHRI.

\section{References}

[1] Z. Q. Du, L. N. Zhang, Q. Lu et al., "Clinical charateristics and outcome of pyogenic liver abscess with different size: 15 -year experience from a single center," Scientific Reports, vol. 6, p. $35890,2016$.

[2] E. R. Lederman and N. F. Crum, "Pyogenic liver abscess with a focus on Klebsiella pneumoniae as a primary pathogen: an emerging disease with unique clinical characteristics," The American Journal of Gastroenterology, vol. 100, no. 2, pp. 322-331, 2005.

[3] L. Meddings, R. P. Myers, J. Hubbard et al., "A populationbased study of pyogenic liver abscesses in the United States: incidence, mortality, and temporal trends," American Journal of Gastroenterology, vol. 105, no. 1, pp. 117-124, 2010.

[4] C.-W. Tseng, Y.-T. Chen, C.-L. Lin, and J.-A. Liang, "Association between chronic pancreatitis and pyogenic liver abscess: a nationwide population study," Current Medical Research and Opinion, vol. 33, no. 3, pp. 505-510, 2017. 
[5] S. W. Lai, C. L. Lin, and K. F. Liao, "Use of oral corticosteroids and risk of hip fracture in the elderly in a case-control study," Frontiers in Pharmacology, vol. 8, p. 625, 2017.

[6] S.-W. Lai, C.-L. Lin, and K.-F. Liao, "Association between oral corticosteroid use and pyogenic liver abscesses in a casecontrol study," BioMedicine, vol. 8, no. 1, p. 5, 2018.

[7] R. H. Straub, M. Cutolo, and R. Pacifici, "Evolutionary medicine and bone loss in chronic inflammatory diseases-a theory of inflammation-related osteopenia," Seminars in Arthritis and Rheumatism, vol. 45, no. 2, pp. 220-228, 2015.

[8] C. C. Hsu, H. C. Hsu, C. C. Lin et al., "Increased risk for hip fractures among patients with cholangitis: a nationwide population-based study," BioMed Research International, vol. 2018, Article ID 8928174, 6 pages, 2018.

[9] Y. C. Chiu, P. S. Liao, Y. T. Chou et al., "The incidence and risk factors of hip fracture after liver transplantation (LT): a nationwide population-based study," BioMed Research International, vol. 2019, Article ID 5845709, 5 pages, 2019.

[10] H. Suzuki, "Bone complications in chronic liver disease (hepatic osteodystrophy)," Clinical Calcium, vol. 25, no. 11, pp. 1619-1624, 2015.

[11] P. Angulo, G. A. Grandison, D. G. Fong et al., "Bone disease in patients with primary sclerosing cholangitis," Gastroenterology, vol. 140, no. 1, pp. 180-188, 2011.

[12] S. Pouwels, A. Lalmohamed, B. Leufkens et al., "Risk of hip/ femur fracture after stroke: a population-based case-control study," Stroke, vol. 40, no. 10, pp. 3281-3285, 2009.

[13] A. M. Alem, D. J. Sherrard, D. L. Gillen et al., "Increased risk of hip fracture among patients with end-stage renal disease," Kidney International, vol. 58, no. 1, pp. 396-399, 2000.

[14] L. Luan, R. Li, Z. Wang et al., "Stroke increases the risk of hip fracture: a systematic review and meta-analysis," Osteoporosis International, vol. 27, no. 11, pp. 3149-3154, 2016. 